\title{
Effet de l'irrigation déficitaire contrôlée sur la croissance et le développement foliaire du palmier dattier (Phoenix dactylifera L.)
}

\author{
Ahmed Sabri ${ }^{1, *}$, Ahmed Bouaziz ${ }^{2}$, Ali Hammani ${ }^{2}$, Marcel Kuper ${ }^{3}$, Ahmed Douaik ${ }^{1}$ \\ et Mohamed Badraoui ${ }^{1,2}$ \\ ${ }^{1}$ Institut national de la recherche agronomique, Rabat, Maroc \\ 2 Institut agronomique et véterinaire Hassan II, Rabat, Maroc \\ ${ }^{3}$ CIRAD, UMR G-Eau, 34398 Montpellier, France
}

\begin{abstract}
Résumé - L'eau est un facteur limitant pour le développement de l'agriculture oasienne. L'amélioration de son efficience d'utilisation est donc nécessaire et peut passer par des stratégies d'irrigation déficitaire. La réponse du palmier dattier à de telles stratégies a ainsi été évaluée par l'étude expérimentale de leurs effets sur la croissance végétative des palmiers adultes de la variété Majhoul. L'expérimentation a été conduite au Tafilalet (Maroc) pendant deux années consécutives (mars 2012-février 2014). Le cycle annuel de la croissance et du développement de cette variété a été découpé en trois périodes distinctes : la première de novembre à février, la seconde de mars à juin et la dernière de juillet à octobre. Pendant ces périodes, sept régimes hydriques ont été appliqués sous irrigation localisée: régime agriculteur (T0), $100 \%$ évapotranspiration maximale [ETM] (T1), $80 \%$ (T2), $60 \%$ (T3), 80-100-60\% (T4), $150 \%$ (T5) et 60$100-80 \%$ (T6). Les mesures ont porté sur le suivi des paramètres météorologiques, les apports d'eau et l'évolution de la biomasse végétative (émission, croissance et sénescence des palmes, et le suivi de l'indice foliaire). Les résultats obtenus montrent que: le régime hydrique et la périodisation affectent significativement la phénologie du palmier dattier en deuxième année; les apports en eau d'irrigation sont en moyenne de $51 \mathrm{~m}^{3} / \mathrm{pied} / \mathrm{an}$, variables entre 30 et $76 \mathrm{~m}^{3} / \mathrm{pied} / \mathrm{an}$; le T6, l'irrigation déficitaire régulée à $60-100-80 \%$ de l'ETM selon les saisons, a amélioré la productivité végétative du palmier et a permis la conservation de $14 \%$ de l'eau d'irrigation comparativement au T1. La stratégie de l'irrigation déficitaire régulée pourrait donc contribuer à la rationalisation de l'eau et à la durabilité des oasis.
\end{abstract}

Mots clés : stratégies de l'irrigation déficitaire / palmier dattier / Majhoul / indice foliaire

Abstract - Effect of regulated deficit irrigation on growth and development of leaf date palm (Phoenix dactylifera L.). Water is a limiting factor for the development of oasis agriculture. Water use efficiency needs to be improved by searching for different strategies of deficit irrigation. In this context, the response of date palm to these strategies was evaluated by the experimental study of the effects on the vegetative growth of mature palms cv Majhoul. The experiment was conducted in Tafilalet (Morocco) for two consecutive years (March 2012-February 2014). The annual cycle of growth and development of this variety was divided into three distinct periods: the first from November to February, the second from March to June, and the last from July to October. During these times, seven water regimes were applied under drip irrigation: farmer regime (T0), 100\% (T1), 80\% (T2), 60\% (T3), 80-100-60\% (T4), 150\% (T5), and 60-100-80\% (T6) ETM. The measures focused on the monitoring of meteorological parameters, the water irrigation and the evolution of vegetative biomass (emission growth and senescence leaves, and monitoring LAI [Leaf Area Index]) of this variety. The results at the end of the second year of this trial show that: water regime and periodization significantly affected the phenology of the date palm in the second year; water irrigation needs are, on average, $51 \mathrm{~m}^{3} /$ tree/year, varying between 30 and $76 \mathrm{~m}^{3} /$ tree/year; the average production of palm and leaf area index were 17.8, 21.1, 20.3, 19.4, 20.1, 20.5, and $21.4 \mathrm{palm} /$ year/tree and 1.01, 1.26, 1.26, 1.22, 1.26, 1.25 and 1.37 , respectively for treatments $\mathrm{T} 0, \mathrm{~T} 1, \mathrm{~T} 2, \mathrm{~T} 3, \mathrm{~T} 4, \mathrm{~T} 5$, and $\mathrm{T} 6$. Thus, the $\mathrm{T} 6$ water regime (regulated deficit irrigation) improved the vegetative growth of date palm and allowed the conservation of

\footnotetext{
* Auteur de correspondance : sabri_inra2004@yahoo.fr; sabri.inra2004@gmail.com
} 
$14 \%$ of irrigation water compared to $\mathrm{T} 1$. The strategy of regulated deficit irrigation could contribute to the rationalization of water and the sustainability of oases.

Keywords: strategies of deficit irrigation / date palm / Majhoul / leaf area index

\section{Introduction}

La palmeraie marocaine compte environ 5 millions de palmiers dattiers dont près de $60 \%$ sont productifs. Ce patrimoine est réparti sur plus de 90 palmeraies, principalement localisées dans les régions de Draâ (1,9 millions de palmiers), du Ziz (1,6 millions de palmiers) et de Tata ( 0,9 millions de palmiers). Celles-ci représentent, à elles seules, $88 \%$ de l'effectif total (MAPM, 2014). Les oasis de Tafilalet regroupent 1,6 millions de palmiers, soit $29 \%$ de l'effectif national (MAPM, 2014). Le climat de cette zone se caractérise par des précipitations faibles et irrégulières et par la succession récente d'années de sécheresse. Ces conditions font des eaux souterraines la source principale pour satisfaire les besoins en eau des palmeraies, notamment les extensions situées à l'extérieur des oasis traditionnelles.

Face à ces contraintes hydriques, l'économie de l'eau d'irrigation constitue un axe incontournable pour la subsistance et la durabilité de ces palmeraies. Les stratégies de l'irrigation déficitaire sont l'un des moyens permettant une meilleure utilisation de l'eau d'irrigation dans les zones à ressources hydriques limitées. Ces stratégies, abondamment étudiées en arboriculture fruitière (English et Raja, 1996), englobent deux types, l'irrigation déficitaire continue et l'irrigation déficitaire régulée. Ce dernier concept a été proposé par Chalmers et al. (1981) pour contrôler la croissance végétative des vergers de pêcher où ils ont constaté qu'une économie d'eau d'irrigation pouvait être réalisée sans réduire le rendement. Des résultats similaires ont été rapportés pour d'autres espèces fruitières dont les études réalisées chez le poirier (Zhao et al., 2015), l'amandier (Razouk et al., 2013a), le mandarinier (Pedrero et al., 2014), le clémentinier (Ballester et al., 2014) et l'abricotier (Pérez-Pastor et al., 2014).

Les études traitant les effets de l'irrigation déficitaire sur la croissance végétative, le développement et le rendement du palmier dattier sont limitées et rares. Ismail et al. (2014) ont étudié, en Arabie Saoudite, l'effet de quatre régimes hydriques (50, 65, 82 et $100 \%$ des besoins en eau du palmier) sur l'efficience de l'utilisation de l'eau d'irrigation (water use efficiency, WUE), le rendement et la qualité des dattes de la variété « Nabbut-Saif ». Ces auteurs ont conclu que le régime $65 \%$ a permis de maximiser le rendement des dattes par la production de $46,1 \mathrm{~kg}$ par arbre et a abouti à la meilleure WUE $\left(1,36 \mathrm{~kg} / \mathrm{m}^{3} /\right.$ arbre $)$, tandis que les régimes hydriques 82 et $100 \%$ des besoins totaux de l'eau ont permis de produire 45,5 et $46 \mathrm{~kg}$ par arbre, respectivement, et ont réduit la WUE. D'autre part, Mazahrih et al. (2012) ont conduit une expérimentation en Jordanie afin de déterminer les coefficients culturaux $(K c)$ et les besoins en eau du palmier dattier cv Majhoul (ETc) sous quatre régimes hydriques (50, 75, 100 et $125 \% \mathrm{ETc})$. Ils ont rapporté que les rendements obtenus étaient de 33,46, 35,98, 37,8 et 42,49 kg/pied et la productivité de l'eau a été de $1,25,0,90,0,71$ et $0,64 \mathrm{~kg} / \mathrm{m}^{3}$, respectivement pour $50,75,100$ et $125 \%$ ETc et que les deux régimes les plus stressés (50 et $75 \%$ ETc) ont par ailleurs augmenté significativement la salinité du sol de 1,5 à $3,5 \mathrm{dS} / \mathrm{m}$.
On note donc un manque considérable de caractérisation des impacts du déficit hydrique sur les principaux paramètres de fonctionnement du palmier dattier. Ainsi, Carr (2012) a recommandé l'étude détaillée de cette stratégie de l'irrigation pour approfondir les observations citées par plusieurs auteurs. La présente étude vise essentiellement à :

- évaluer expérimentalement l'effet du stress hydrique contrôlé sur la croissance et le développement de la biomasse végétative du palmier dattier adulte;

- identifier les phases sensibles au sein du cycle annuel du palmier dattier.

\section{Matériels et méthodes}

\subsection{Site expérimental}

L'expérimentation a été conduite pendant deux années consécutives (mars 2012-février 2014), au sein d'une exploitation privée de 14 hectares, située dans la zone de Goulmima dans la province d'Errachidia, à environ $500 \mathrm{~km}$ de Rabat dans le Sud-Est Marocain (longitude 4'54'37' Ouest, latitude $31^{\circ} 41$ ' 11 " Nord, altitude de $1000 \mathrm{~m}$ ). Le climat, de type subdésertique sec et continental, est caractérisé par un été chaud et un hiver froid. La température maximale est de $43^{\circ} \mathrm{C}$ et la température minimale est de $-3{ }^{\circ} \mathrm{C}$. Les pluviométries sont faibles et irrégulières dans le temps et dans l'espace. Elles varient entre 11 et $293 \mathrm{~mm} / \mathrm{an}$ avec une moyenne de $116 \mathrm{~mm} /$ an durant une période de 32 ans, allant de 1979/80 jusqu'à 2010/2011 (ORMVA/TF, 2012).

L'analyse physique du sol du site expérimental sur une profondeur de $180 \mathrm{~cm}$, montre que la texture du sol de cette parcelle est variable et va, selon le diagramme de classification des textures de Danis (2000), d'une texture argileuse à limono-argilo-sableuse, avec une dominance de cette dernière ( $2 / 3$ des échantillons). Le taux des limons est relativement faible avec prédominance des taux inférieurs à $20 \%$.

Excepté dans les premiers 20 centimètres de profondeur, la conductivité électrique de la solution du sol est inférieure à $2 \mathrm{mmhos} / \mathrm{cm}$, valeur pour laquelle aucune baisse des rendements n'est à craindre (FAO, 1980).

\subsection{Matériel végétal}

Le cultivar choisi est la variété Majhoul. Cette variété se caractérise essentiellement par sa notoriété mondiale liée à la qualité de ses dattes, engendrant une valeur ajoutée commerciale.

Les palmiers mis en essai ont été plantés en 2000, à une densité de 156 pieds/ha $(8 \mathrm{~m} \times 8 \mathrm{~m})$. Ils ont au moment de l'essai des stipes d'une hauteur moyenne de $2 \mathrm{~m}$ et sont dépourvus de tout signe de carence ou de maladie. La superficie réservée à l'essai couvre environ un hectare, y compris les bordures et les servitudes. 
Tableau 1. Signification des régimes hydriques étudiés. Table 1. Meaning of the studied water regimes.

\begin{tabular}{lllll}
\hline Traitements & $\begin{array}{l}\text { Phase I } \\
\text { (novembre à } \\
\text { février) }\end{array}$ & $\begin{array}{l}\text { Phase II } \\
\text { (mars à } \\
\text { juin) }\end{array}$ & $\begin{array}{l}\text { Phase III } \\
\text { (juillet à } \\
\text { octobre) }\end{array}$ \\
\hline $\mathrm{T}_{0}$ & $\mathrm{~T}$ Tag & $\begin{array}{l}\text { Régime } \\
\text { agriculteur }\end{array}$ & $\begin{array}{l}\text { Régime } \\
\text { agriculteur }\end{array}$ & $\begin{array}{l}\text { Régime } \\
\text { agriculteur }\end{array}$ \\
$\mathrm{T}_{1}$ & $\mathrm{~T}_{100-100-100}$ & $100 \%$ ETM & $100 \%$ ETM & $100 \%$ ETM \\
$\mathrm{T}_{2}$ & $\mathrm{~T}_{80-80-80}$ & $80 \%$ ETM & $80 \%$ ETM & $80 \%$ ETM \\
$\mathrm{T}_{3}$ & $\mathrm{~T}_{60-60-60}$ & $60 \%$ ETM & $60 \%$ ETM & $60 \%$ ETM \\
$\mathrm{T}_{4}$ & $\mathrm{~T}_{80-100-60}$ & $80 \%$ ETM & $100 \%$ ETM & $60 \%$ ETM \\
$\mathrm{T}_{5}$ & $\mathrm{~T}_{150-150-150}$ & $150 \%$ ETM & $150 \%$ ETM & $150 \%$ ETM \\
$\mathrm{T}_{6}$ & $\mathrm{~T}_{60-100-80}$ & $60 \%$ ETM & $100 \%$ ETM & $80 \%$ ETM \\
\hline
\end{tabular}

NB : ETM : évapotranspiration maximale.

\subsection{Dispositif expérimental}

Les 84 palmiers utiles, inclus dans l'essai, sont répartis en 21 unités expérimentales (comportant chacune quatre palmiers) suivant un dispositif en blocs aléatoires complets avec trois répétitions. Chaque groupe de 12 arbres a été soumis à un régime hydrique variant entre 60 et $150 \%$ de l'évapotranspiration maximale [ETM] (Tab. 1).

Les sept régimes hydriques étudiés sont répartis de la manière suivante :

- $\mathrm{T}_{0}$ est le régime hydrique adopté par l'agriculteur pour arroser ses palmiers; c'est la référence locale;

- $\mathrm{T}_{1}$ correspond à $100 \%$ de l'ETM équivalent aux besoins en eau du palmier dattier, et représente le témoin dans cette étude;

- $T_{2}$ et $T_{3}$ sont les deux régimes hydriques qui correspondent aux irrigations déficitaires continues aux seuils de déficit hydrique de respectivement 20 et $40 \%$, soit 80 et $60 \%$ de l'ETM durant toute l'année;

- Les régimes hydriques $T_{4}$ et $T_{6}$ correspondent aux irrigations déficitaires contrôlées ou régulées aux seuils de déficit hydrique de, respectivement, 20 et $40 \%$ de l'ETM durant la phase hivernale (novembre-février), et 40 et $20 \%$ de l'ETM pendant la période estivale (juilletoctobre), soit $80-100-60$ et $60-100-80 \%$ de l'ETM;

- $\mathrm{T}_{5}$ est le régime hydrique qui assure un apport d'eau qui dépasse toujours les besoins en eau de l'ETM de $50 \%$, et ce durant toute l'année (soit $150 \%$ de l'ETM).

Le découpage en trois périodes distinctes est raisonné conformément aux phases du cycle annuel du palmier dattier, à savoir:

- la première période qui s'étale de novembre à février, juste après la récolte où la plante passe par une période de repos végétatif;

- la seconde s'étalant de mars à juin, durant laquelle se déroulent la pollinisation, la croissance et le grossissement des fruits et la croissance active du palmier dattier;

- la dernière période allant de juillet à octobre et qui correspond aux phases de fin de grossissement des fruits, croissance active du palmier dattier et maturation des fruits (Sedra, 2003, 2012).
L'irrigation localisée est assurée par un système en goutte à goutte aménagé en couronnes autour des palmiers. Chaque arbre est entouré d'une couronne dont le diamètre est d'environ $3 \mathrm{~m}$, comprenant six goutteurs boutons espacés de $150 \mathrm{~cm}$, et débitant chacun $25 \mathrm{l} / \mathrm{h}$ pour un débit total de $150 \mathrm{l} / \mathrm{h}$.

La fréquence d'irrigation varie entre deux et sept fois par semaine en fonction de la demande climatique. Elle est de deux, trois et sept fois par semaine respectivement durant les mois de novembre à février, mars et octobre, et d'avril à septembre.

Les doses apportées par irrigation varient en fonction des traitements hydriques et des saisons. Pour le cas du témoin (100\% de l'ETM), la dose apportée par irrigation varie entre 136 et 242 1/pied/irrigation, soit l'équivalent à l'échelle de l'hectare de 20 et $33 \mathrm{~m}^{3} /$ ha/irrigation. Pour le cas du $\mathrm{T}_{0}$, ce paramètre varie entre 240 et $400 \mathrm{l} / \mathrm{pied} /$ irrigation, soit l'équivalent de 33 et $55 \mathrm{~m}^{3} / \mathrm{ha} /$ irrigation à l'échelle de l'hectare.

\subsection{Estimation des besoins en eau d'irrigation}

\subsubsection{Calcul de l'évapotranspiration de référence}

Le calcul de l'évapotranspiration de référence $\mathrm{ET}_{0}$ a été effectué selon la formule de Penman-Monteith (Allen et al., 1998) en utilisant les paramètres météorologiques de la station agro-météorologique située sur l'exploitation.

\subsection{2 Évaluation de l'évapotranspiration maximale du palmier}

L'évaluation des besoins en eau du palmier (en $1 /$ arbre/j) est donnée par la formule suivante (Gafar, 2010 ; Ismail et al., 2014).

$$
E T M_{\text {palmier }}=E T_{0} \times K_{c} \times S_{a} .
$$

Les coefficients culturaux $K c$ utilisés sont les $K c$ de la FAO rapportés par Liebenberg et Zaid (2002) et qui ont été utilisés à Naute en Namibie. Ces coefficients culturaux ont varié entre 0,8 et 1 en fonction des phases du cycle annuel du palmier dattier.

$S_{a}$ correspond à l'aire active de l'évapotranspiration du palmier $\left(\mathrm{m}^{2}\right)$, elle est obtenue suivant la formule ci-après où le rayon actif $\left(R_{a}\right)$ est égal à $90 \%$ du rayon de la surface de la projection verticale de la frondaison $(\mathrm{m})$.

$$
S_{a}=\pi\left(R_{a}\right)^{2} .
$$

\subsubsection{Détermination du besoin brut en eau d'irrigation}

Le besoin net $(B n)$ en eau d'irrigation $(1 /$ plant $/ j)$ est donné par l'équation suivante :

$$
B n\left(\frac{l / \text { plant }}{j}\right)=\frac{\operatorname{ETM}\left(\frac{l \text { plant }}{j}\right)}{1-\mathrm{LR}} .
$$

$L R$ est le besoin de lessivage ou la fraction de l'eau d'irrigation qui doit traverser la zone racinaire active pour maintenir la salinité à un niveau déterminé. Dans le cas de 
l'irrigation localisée, ce besoin est calculé à l'aide de la formule suivante (Doorenbos et Pruitt, 1977) :

$$
L R=\frac{E C_{W}}{2{\operatorname{Max} E C_{e}}_{e}} .
$$

$E C_{w}$ est la conductivité électrique de l'eau d'irrigation, $\operatorname{Max} E C_{e}$ représente la valeur maximum tolérable de la conductivité électrique de l'extrait du sol saturé en mmhos/ $\mathrm{cm}$. Dans le cas de cet essai, $E C_{w}=4,03 \mathrm{mmhos} / \mathrm{cm}$ et $M a x E C_{e}$ est de $32 \mathrm{mmhos} / \mathrm{cm}$ (Doorenbos and Pruitt, 1977).

\subsubsection{Détermination du besoin brut en eau d'irrigation}

Le besoin brut en eau d'irrigation, noté $B b$, est défini par:

$$
B b\left(\frac{l / \text { plant }}{\mathrm{j}}\right)=\frac{B n\left(\frac{l / \text { plant }}{j}\right)}{E_{a}},
$$

avec $E_{a}$ est l'efficience de l'irrigation à la parcelle. Dans le cas de cet essai, l' $E_{a}$ calculée est égale à $90 \%$.

\subsection{Paramètres de croissance foliaire observés}

Dans le présent travail, pour quantifier et évaluer l'effet du régime hydrique sur la croissance, le développement du feuillage du palmier dattier, le contrôle et le suivi des différents paramètres liés à ces processus ont été effectués à des intervalles précis (Tab. 2). À signaler aussi que sur la même parcelle expérimentale, l'évaluation de l'effet du stress hydrique contrôlé sur le rendement en dattes et sur la valorisation de l'eau par le dattier a été étudiée et que le détail relatif à ces aspects figure dans Sabri et al. (2017).

La surface foliaire d'une palme (SFF) $\left(\mathrm{m}^{2}\right)$ a été estimée grâce à la relation qui existe entre cette surface et le produit de la longueur de la feuille (LF) (m) et celle de la foliole médiane (LFM) (m) (Ben Henia, 2012). La surface totale de la biomasse végétale (SFP) du palmier $\left(\mathrm{m}^{2}\right)$ est obtenue donc en multipliant la SFF par le nombre total de palmes actives (NPA) en un moment donné.

$$
\begin{gathered}
S F F=0,683 \times(L F \times L F M)^{1,018} . \\
S F P\left(m^{2}\right)=N P A \times S F F\left(m^{2}\right) .
\end{gathered}
$$

L'indice foliaire (LAI) a été calculé en divisant la surface foliaire du palmier (SFP) par la surface au sol d'un palmier $\left(\mathrm{S} 1 \mathrm{P}=64 \mathrm{~m}^{2}\right.$, avec cette densité de plantation).

$$
\operatorname{LAI}\left(m^{2} / m^{2}\right)=\frac{S F P\left(m^{2}\right)}{S 1 P\left(m^{2}\right)} .
$$

\subsection{Analyses statistiques}

Le dépouillement des données des paramètres étudiés ainsi que le calcul des moyennes et la conception des graphiques ont été réalisés à l'aide du tableur Excel. L'analyse de la variance (ANOVA) a été effectuée en utilisant la procédure GLM du logiciel statistique SAS. Deux facteurs de classification ont été utilisés, la dose d'irrigation et la période. Chaque fois que l'effet de l'un de ces facteurs ou leurs interactions s'est montré
Tableau 2. Nature des paramètres contrôlés et fréquence des observations.

Table 2. Nature of the controlled parameters and frequency of observations.

\begin{tabular}{ll}
\hline Nature des paramètres contrôlés & Fréquence des observations \\
\hline $\begin{array}{l}\text { Nombre de palmes émises } \\
\text { Nombre de palmes desséchées }\end{array}$ & $\begin{array}{l}\text { À la fin de chaque période } \\
\text { À la fin de chaque période } \\
\text { de la foliole médiane }\end{array}$ \\
$\begin{array}{l}\text { Rayon de la surface de projection } \\
\text { de la frondaison }\end{array}$ & Une fois par an \\
$\begin{array}{l}\text { Surface foliaire } \\
\text { Indice de la surface foliaire }\end{array}$ & À la fin de chaque période \\
\hline
\end{tabular}

Tableau 3. Bilan des apports de l'eau d'irrigation $\left(\mathrm{m}^{3} / \mathrm{pied} / \mathrm{an}\right)$. Table 3. Assessment of water irrigation $\left(\mathrm{m}^{3} /\right.$ tree/year $)$.

\begin{tabular}{llll}
\hline Traitements & $1^{\text {er }}$ année & $2^{\text {e }}$ année & Année moyenne \\
\hline T0 & 69,61 & 69,61 & 69,61 \\
T1 & 48,69 & 52,19 & 50,44 \\
T2 & 38,95 & 41,75 & 40,35 \\
T3 & 29,21 & 31,31 & 30,26 \\
T4 & 38,86 & 42,12 & 40,49 \\
T5 & 73,03 & 78,29 & 75,66 \\
T6 & 41,85 & 45,35 & 43,60 \\
\hline
\end{tabular}

significatif, on a procédé à une comparaison multiple des moyennes à l'aide des tests de Duncan et de Dunnett à un seuil de $5 \%$.

\section{Résultats et discussions}

\subsection{Bilan des besoins en eau d'irrigation du palmier dattier}

Durant les deux années de conduite de l'expérimentation, le volume total des apports d'eau correspondant aux besoins en eau du palmier dattier a varié entre 48 et $52 \mathrm{~m}^{3} /$ arbre/an, avec une moyenne de $50,4 \mathrm{~m}^{3} /$ arbre/an, soit un besoin annuel moyen par hectare de l'ordre de $7870 \mathrm{~m}^{3} / \mathrm{ha} /$ an (Tab. 3). Le détail relatif à ces aspects figure dans Sabri et al. (2017).

L'application des régimes hydriques, décrits dans la méthodologie (Sect. 2.3), a permis de totaliser les volumes d'eau apportés durant la conduite de l'expérimentation. Le Tableau 3 fait ressortir les volumes totaux apportés par traitement et par année. Ces volumes ont varié entre 29 et $73 \mathrm{~m}^{3}$ /arbre en 2012 et entre 31 et $78 \mathrm{~m}^{3}$ /arbre/an en deuxième année. La moyenne, toutes années confondues, varie entre 30,3 et $75,7 \mathrm{~m}^{3} / \mathrm{pied} / \mathrm{an}$.

\subsection{Effet du stress hydrique sur l'évolution du feuillage du palmier dattier}

\subsection{1 Évolution de l'émission des palmes}

L'évaluation de l'effet du régime hydrique sur la vitesse d'émission des feuilles observée à la fin de la première année 


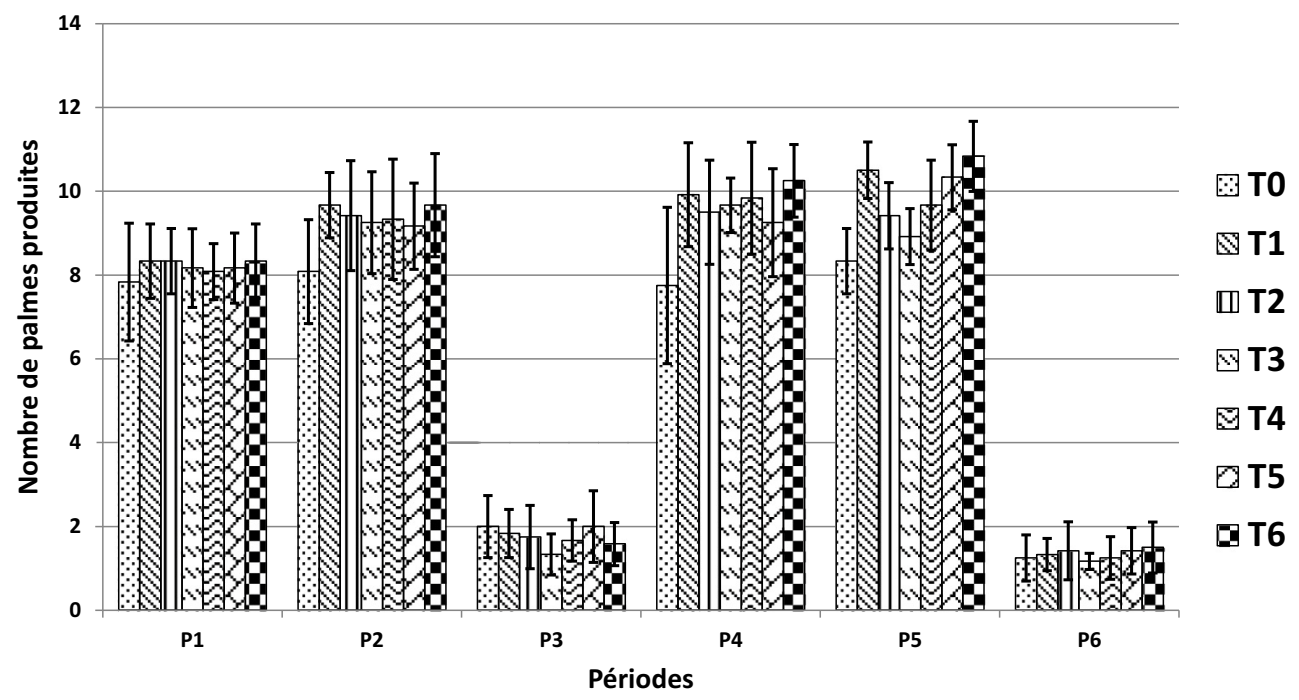

Fig. 1. Effet du régime hydrique sur l'évolution de l'émission des palmes; les barres représentent les erreurs standards.

Fig. 1. Effect of water regime on the evolution of the issue of palms; bars representing standard errors.

Tableau 4. Effet cumulatif du régime hydrique sur la vitesse d'émission des palmes.

Table 4. Cumulative effect of water regime on the transmission speed of palms.

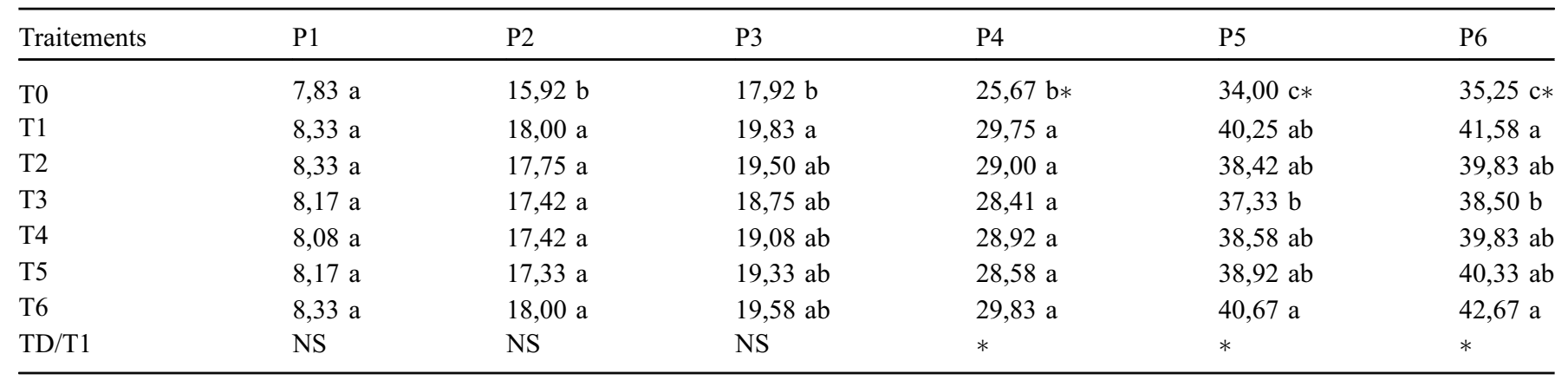

La même lettre dans la même colonne indique la non-signification des différences entre les traitements selon le test de Duncan.

TD/T1 : Test de Dunnett en prenant T1 comme référence (NS : non significatif; $*$ : significatif).

P1 : période mars-juin 2012; P2 : Juillet-octobre 2012 ; P3 : Novembre 2012-février 2013 ; P4 : Mars-juin 2013 ; P5 : Juillet-octobre 2013 ; P6 :

Novembre 2013-février 2014, Ces périodes sont les mêmes pour les autres tableaux.

de l'application des régimes hydriques (Fig. 1) montre que, généralement, les sept régimes hydriques (T0 à T6) ont évolué presque de la même manière et que les écarts entre eux étaient relativement faibles. En effet, les palmiers, mis en essai, ont produit en moyenne par ordre décroissant 19,8, 19,6, 19,5, $19,3,19,1,18,8$ et $17,9 \mathrm{palmes} / \mathrm{an} / \mathrm{pied}$, respectivement pour les traitements T1, T6, T2, T5, T4, T3 et T0.

En deuxième année, l'observation du même paramètre montre des changements au niveau du classement. Ainsi, les mêmes palmiers ont formé en moyenne par ordre décroissant $22,6,21,8,21,20,8,20,3,19,8$ et 17,3 palmes/an /pied, respectivement pour les traitements T6, T1, T5, T4, T2, T3 et T0.

L'analyse statistique de la vitesse d'émission des palmes par période, à un seuil de probabilité de $5 \%$, en considérant chaque période à part, montre que les écarts non-significatifs apparaissent à partir de la deuxième période (juillet-octobre
2012). C'est uniquement lors de la même période en 2013 que nous avons enregistré des différences significatives entre les traitements. Le test de Duncan, au même seuil de probabilité, a réparti les traitements en cinq groupes avec des chevauchements entre les groupes (Fig. 1).

Par contre, si on considère l'effet cumulatif du régime hydrique sur le développement des feuilles, on note aussi que les différences non-significatives apparaissent à partir de la deuxième période 2012 (juillet-octobre 2012), même si le test du Dunnett montre que T0 est différent de T1. Ces différences deviennent significatives à partir de la première période de 2013 et ce jusqu'à la fin de l'essai (Tab. 4).

Il en résulte donc que le palmier dattier est plus actif et productif en période estivale, de juillet à octobre. L'effet de cette période est remarquable, notamment en deuxième année, et a affecté significativement l'émission des palmes. 
Tableau 5. Effet cumulatif du régime hydrique sur le nombre de palmes actives.

Table 5. Cumulative effect of water regime on the number of active leaves.

\begin{tabular}{llllllll}
\hline Traitements & P0 & P1 & P2 & P3 & P4 & P5 \\
\hline T0 & $55,00 \mathrm{~b}$ & $58,00 \mathrm{~b}$ & $56,67 \mathrm{~b} *$ & $58,67 \mathrm{~b} *$ & $60,67 \mathrm{~b} *$ & $60,75 \mathrm{c} *$ & $62,00 \mathrm{c} *$ \\
T1 & $60,00 \mathrm{ab}$ & $63,83 \mathrm{a}$ & $66,08 \mathrm{a}$ & $67,92 \mathrm{a}$ & $72,58 \mathrm{a}$ & $75,58 \mathrm{ab}$ \\
T2 & $59,17 \mathrm{ab}$ & $62,75 \mathrm{ab}$ & $65,17 \mathrm{a}$ & $66,92 \mathrm{a}$ & $71,58 \mathrm{a}$ & $73,92 \mathrm{ab}$ & $75,92 \mathrm{ab}$ \\
T3 & $60,42 \mathrm{ab}$ & $64,75 \mathrm{a}$ & $64,58 \mathrm{a}$ & $65,92 \mathrm{a}$ & $71,58 \mathrm{a}$ & $70,67 \mathrm{~b}$ \\
T4 & $61,25 \mathrm{a}$ & $65,92 \mathrm{a}$ & $67,33 \mathrm{a}$ & $69,00 \mathrm{a}$ & $75,08 \mathrm{a}$ & $76,17 \mathrm{ab}$ & $77,83 \mathrm{~b}$ \\
T5 & $60,42 \mathrm{ab}$ & $64,17 \mathrm{a}$ & $66,33 \mathrm{a}$ & $68,33 \mathrm{a}$ & $72,50 \mathrm{a}$ & $75,50 \mathrm{ab}$ \\
T6 & $63,33 \mathrm{a}$ & $67,00 \mathrm{a}$ & $69,58 \mathrm{a}$ & $71,17 \mathrm{a}$ & $76,42 \mathrm{a}$ & $79,58 \mathrm{a}$ \\
TD/T1 & NS & NS & $*$ & $*$ & $*$ & $*$ \\
\hline
\end{tabular}

La même lettre dans la même colonne indique la non-signification des différences entre les traitements selon le test de Duncan. TD/T1 : Test de Dunnett en prenant T1 comme référence (NS : non significatif; $*$ : significatif).

P0 : début de l'essai (mars 2012).

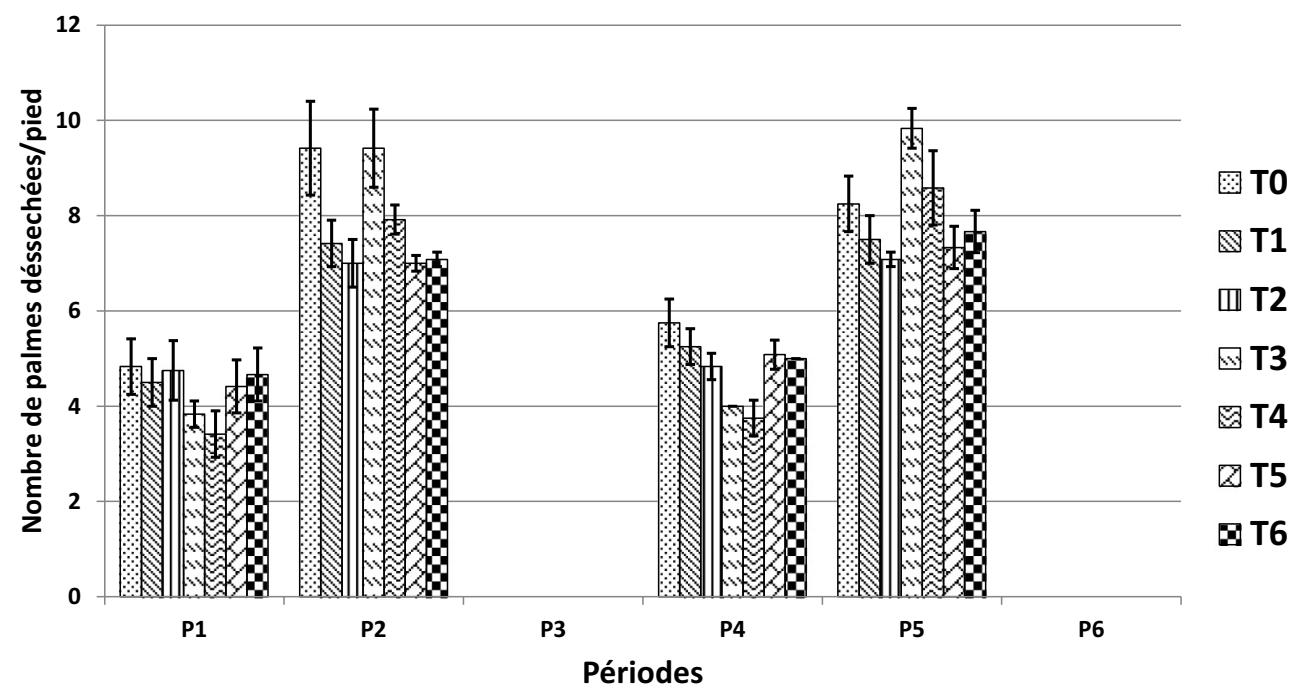

Fig. 2. Effet du régime hydrique sur la vitesse de la sénescence des palmes/pied/période; les barres représentent les écarts moyens.

Fig. 2. Effect of water regime on the rate of senescence palms/tree/period; bars representing average differences.

C'est aussi lors de cette période que le palmier a enregistré des changements et des réactions aux effets du traitement hydrique. Cela explique que cette période estivale corresponde à la période critique et sensible du dattier.

Concernant l'effet du stress hydrique sur l'évolution du feuillage actif (NPA), des différences significatives apparaissent à partir de la deuxième période (juillet-octobre 2012) jusqu'à la fin de l'essai. La comparaison multiple des moyennes, à l'aide du test de Dunnett, montre que seul le T0 est statistiquement différent du T1 (Tab. 5).

\subsection{2 Évolution de la sénescence des palmes}

Contrairement aux observations de plusieurs auteurs (Djerbi, 1988 ; Péreau-Leroy, 1958 ; Sedra, 2003, 2012) relatives à l'égalité de la vitesse annuelle de la sénescence et de la production des palmes, on constate que les palmiers ont perdu, en moyenne, environ $60 \%, 70 \%$ et $80 \%$ de la production annuelle, respectivement pour le premier (T1, T2, T4, T5 et T6), le deuxième (T3) et le dernier (T0) groupe de traitements. Ce constat se retrouve pendant toute la durée de l'essai (Fig. 2).

Les résultats montrent aussi que ce paramètre dépend des périodes (Fig. 2). La vitesse maximale de desséchement des feuilles est enregistrée en période estivale et on note l'absence de ce processus pendant la phase de repos végétatif (toutes années confondues).

L'analyse statistique de la vitesse de sénescence des palmes par période, à un seuil de probabilité de $5 \%$, en considérant chaque période à part, montre des différences significatives entre les traitements. Le test de Duncan groupe les régimes hydriques en plusieurs ensembles avec l'existence parfois de chevauchements entre les groupes.

La comparaison multiple des moyennes faite à l'aide du Test de Dunnett montre que les moyennes des T0 et T3, comparées à celle du $\mathrm{T} 1$, sont significativement différentes uniquement en première année. Excepté le T0 qui a enregistré une augmentation de la sénescence des feuilles, les autres régimes ont produit une diminution relative de ce paramètre. 


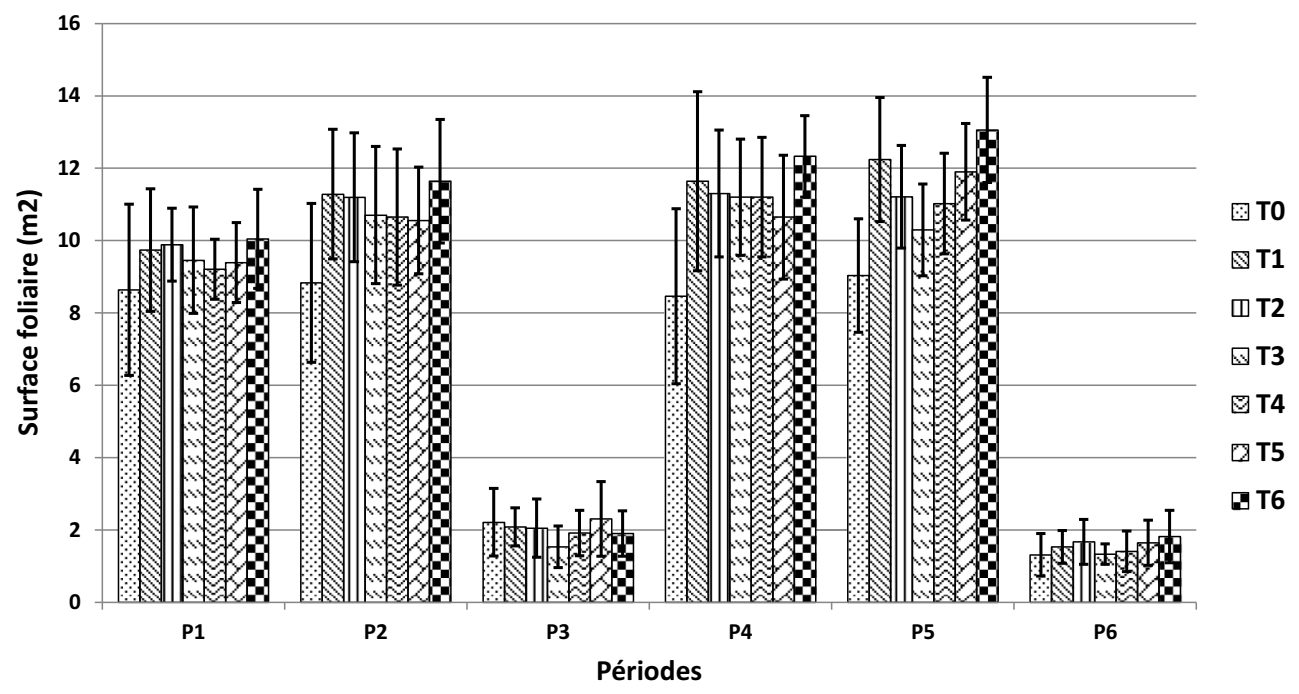

Fig. 3. Effet du régime hydrique sur l'évolution de la surface foliaire produite des palmes $\left(\mathrm{m}^{2} /\right.$ période/pied); les barres représentent les écarts moyens.

Fig. 3. Effect of water regime on the evolution of leaf area produced by palms $\left(\mathrm{m}^{2} /\right.$ period/tree); the bars represent the average differences.

Tableau 6. Évolution périodique de la surface foliaire active $\left(\mathrm{m}^{2} / \mathrm{pied} /\right.$ période) Table 6. Evolution of the periodic active leaf area $\left(\mathrm{m}^{2} /\right.$ tree/ period).

\begin{tabular}{llllllll}
\hline Traitements & P0 & P1 & P2 & P3 & P4 & P5 \\
\hline T0 & $60,37 \mathrm{~b}$ & $63,68 \mathrm{~b}$ & $62,18 \mathrm{~b} *$ & $64,40 \mathrm{~b} *$ & $66,62 \mathrm{~b} *$ & $66,70 \mathrm{c} *$ \\
T1 & $70,43 \mathrm{ab}$ & $74,92 \mathrm{a}$ & $77,57 \mathrm{a}$ & $79,65 \mathrm{a}$ & $85,15 \mathrm{a}$ & $88,62 \mathrm{ab}$ & $90,01 \mathrm{c} *$ \\
T2 & $70,55 \mathrm{ab}$ & $74,81 \mathrm{a}$ & $77,70 \mathrm{a}$ & $79,75 \mathrm{a}$ & $85,30 \mathrm{a}$ & $88,09 \mathrm{ab}$ \\
T3 & $70,26 \mathrm{ab}$ & $75,27 \mathrm{a}$ & $75,10 \mathrm{a}$ & $76,63 \mathrm{a}$ & $83,21 \mathrm{a}$ & $82,13 \mathrm{~b}$ \\
T4 & $70,02 \mathrm{ab}$ & $75,35 \mathrm{a}$ & $76,95 \mathrm{a}$ & $78,87 \mathrm{a}$ & $85,81 \mathrm{a}$ & $87,04 \mathrm{ab}$ \\
T5 & $69,65 \mathrm{ab}$ & $73,95 \mathrm{a}$ & $76,45 \mathrm{a}$ & $78,76 \mathrm{a}$ & $83,56 \mathrm{a}$ & $87,01 \mathrm{ab}$ \\
T6 & $76,32 \mathrm{~b}$ & $80,74 \mathrm{a}$ & $83,85 \mathrm{a}$ & $85,75 \mathrm{a}$ & $92,06 \mathrm{a}$ & $95,88 \mathrm{a}$ \\
TD/T1 & NS & NS & $*$ & $*$ & $*$ & $* 7,66 \mathrm{ab}$ \\
\hline
\end{tabular}

La même lettre dans la même colonne indique la non-signification des différences entre les traitements selon le test de Duncan. TD/T1 : Test de Dunnett en prenant T1 comme référence (NS : non significatif, $*$ : significatif).

\subsection{3 Évolution périodique de la surface foliaire}

Le même constat est fait pour la surface foliaire (Fig. 3). Les différences entre les traitements sont apparues au cours de la deuxième période de la première année et les maxima ont été enregistrés lors de la même période, l'année suivante.

L'analyse statistique de la vitesse d'évolution de la surface foliaire par période, à un seuil de probabilité de $5 \%$, en considérant chaque période à part, montre que les écarts nonsignificatifs apparaissent à partir de la deuxième période (juillet-octobre 2012). En 2013, nous avons enregistré des différences significatives entre les traitements uniquement lors de la quatrième et la cinquième période. Le test de Duncan, au même seuil de probabilité, a réparti les traitements en cinq groupes avec des chevauchements entre les groupes (Fig. 3).

Par contre, si on considère l'effet du régime hydrique sur la vitesse d'évolution de la surface foliaire active par période, nous constatons que les différences significatives apparaissent à partir de la deuxième période de 2012 (juillet-octobre 2012) et restent significatives jusqu'à la fin de l'essai (Tab. 6).

\subsection{4 Évolution de l'indice foliaire}

Généralement, l'allure de l'évolution de l'indice foliaire (leaf area index, LAI) est presque la même pour tous les régimes hydriques (Fig. 4 et Tab. 7). Toutefois, les écarts moyens constatés au départ (début mars 2012), environ 0,04 , ont doublé à la fin (fin février 2014) pour atteindre 0,10 . On remarque aussi que la croissance maximum de LAI a lieu pendant la période printanière. Cela est lié au bilan des processus de développement et de sénescence des feuilles : la majeure partie de la sénescence des feuilles est enregistrée pendant la période estivale, ce qui influe négativement sur la biomasse active pendant cette période. Cette progression du LAI est liée à la stabilité du taux de couverture du sol constaté durant la conduite de l'essai. Cette remarque confirme les résultats de Al-Khafaf et al. (1998) et Gafar (2010) qui rapportent que la projection de la frondaison du palmier adulte stagne à partir de la huitième année de plantation.

D'une manière générale, la progression remarquable du LAI est due essentiellement au bilan positif de la biomasse 


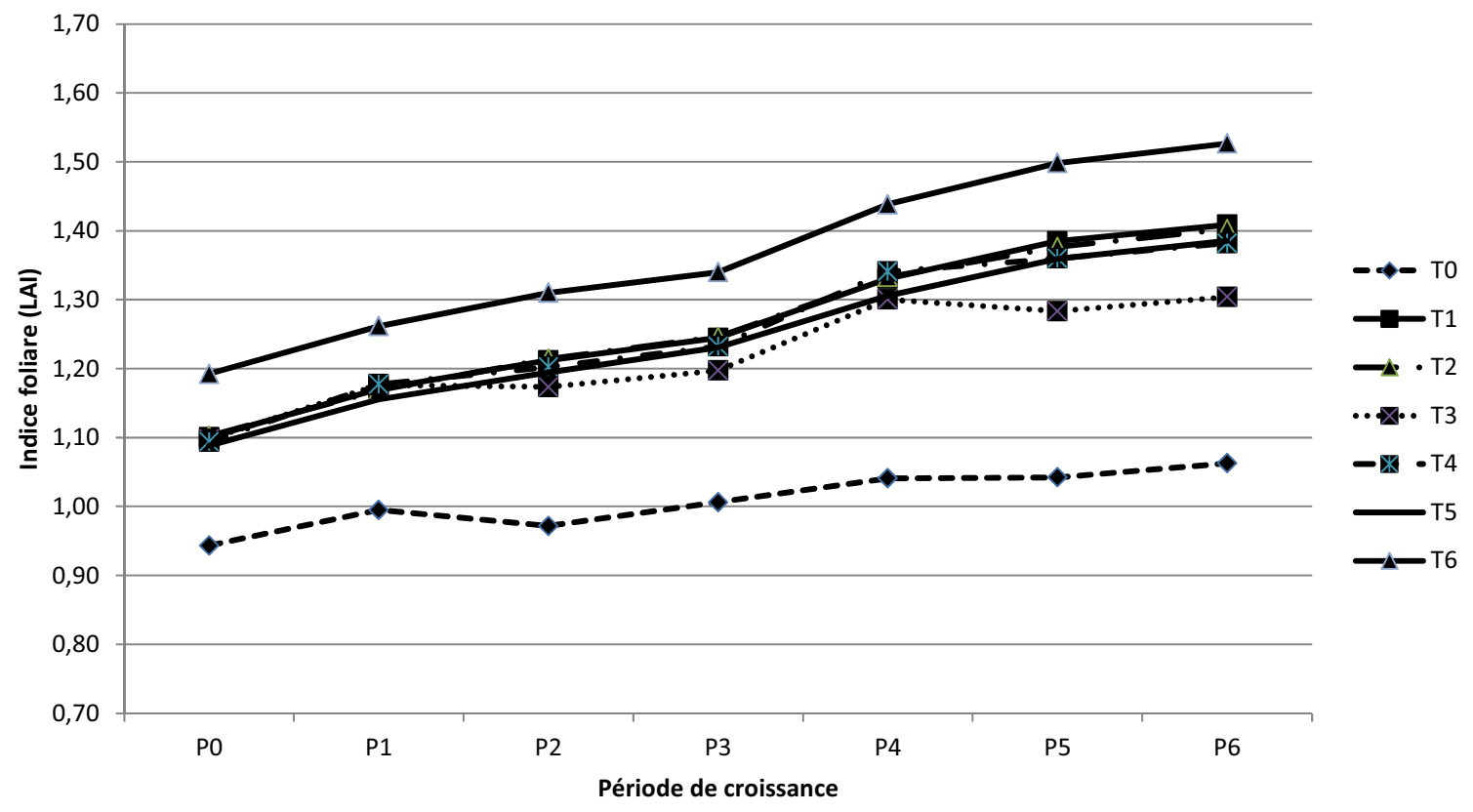

Fig. 4. Effet du régime hydrique sur l'évolution périodique de l'indice foliaire $\left(\mathrm{m}^{2} / \mathrm{m}^{2}\right)$.

Fig. 4. Effect of water regime on the time change in the leaf area index $\left(\mathrm{m}^{2} / \mathrm{m}^{2}\right)$.

Tableau 7. Évolution de l'indice foliaire (LAI) $\left(\mathrm{m}^{2} / \mathrm{m}^{2} / \mathrm{pied} /\right.$ période).

Table 7. Evolution of leaf area index (LAI) $\left(\mathrm{m}^{2} / \mathrm{m}^{2} /\right.$ treel period $)$.

\begin{tabular}{lllllll}
\hline Traitements & P0 & P1 & P2 & P3 & P4 & P5 \\
\hline T0 & $0,94 \mathrm{~b}$ & $1,00 \mathrm{~b}$ & $0,97 \mathrm{~b}$ & $1,01 \mathrm{~b}$ & $1,04 \mathrm{~b} *$ & $1,04 \mathrm{~b} *$ \\
T1 & $1,10 \mathrm{ab}$ & $1,17 \mathrm{ab}$ & $1,21 \mathrm{ab}$ & $1,24 \mathrm{ab}$ & $1,33 \mathrm{a}$ & $1,06 \mathrm{~b} *$ \\
T2 & $1,10 \mathrm{ab}$ & $1,17 \mathrm{ab}$ & $1,21 \mathrm{ab}$ & $1,25 \mathrm{ab}$ & $1,33 \mathrm{a}$ & $1,38 \mathrm{a}$ \\
T3 & $1,10 \mathrm{ab}$ & $1,18 \mathrm{ab}$ & $1,17 \mathrm{ab}$ & $1,20 \mathrm{ab}$ & $1,30 \mathrm{a}$ & $1,28 \mathrm{ab}$ \\
T4 & $1,09 \mathrm{ab}$ & $1,18 \mathrm{ab}$ & $1,20 \mathrm{ab}$ & $1,23 \mathrm{ab}$ & $1,34 \mathrm{a}$ & $1,40 \mathrm{a}$ \\
T5 & $1,09 \mathrm{ab}$ & $1,16 \mathrm{ab}$ & $1,19 \mathrm{ab}$ & $1,23 \mathrm{ab}$ & $1,31 \mathrm{a}$ & $1,36 \mathrm{a}$ \\
T6 & $1,19 \mathrm{a}$ & $1,26 \mathrm{a}$ & $1,31 \mathrm{a}$ & $1,34 \mathrm{a}$ & $1,44 \mathrm{a}$ & $1,30 \mathrm{a}$ \\
TD/T1 & NS & NS & NS & NS & $*$ & $1,39 \mathrm{a}$ \\
\hline Ta & & & $*$ & $*$ \\
\hline
\end{tabular}

La même lettre dans la même colonne indique la non-signification des différences entre les traitements selon le test de Duncan. TD/T1 : Test de Dunnett en prenant T1 comme référence (NS : non significatif, $*$ : significatif).

active régularisée par les processus de développement et de la sénescence des feuilles (Fig. 1 et 2).

Le palmier dattier répond au stress hydrique par la réduction de la biomasse végétative active à travers deux processus simultanés. Le premier est la diminution de la vitesse d'émission de feuilles constituant la surface évapotranspirante. Le second est l'augmentation de la vitesse de sénescence. Ceci afin d'éviter les pertes d'eau à travers la surface exposée. Ce qui se répercute sur l'activité photosynthétique et se traduit finalement par la réduction des rendements. C'est le mécanisme que les végétaux adoptent face au changement des conditions du milieu (Tardieu, 2005). Dans un article sur les rendements de ce même essai (Sabri et al., 2017), nous mettons en évidence une réduction des rendements de l'ordre de $25 \%$ en cas de réduction du régime hydrique de $20 \%$ (traitement $80 \%$ de l'ETM) tout au long de l'année par rapport au traitement témoin $(60,6 \mathrm{~kg} / \mathrm{an} / \mathrm{pied})$. En revanche, quand le déficit hydrique est appliqué de façon différenciée (60\%-100\%-80\% de l'ETM en découpant l'année en trois périodes), la réduction du rendement est négligeable (baisse de 2,4\%). Des résultats similaires ont été obtenus sur d'autres espèces de rosacées fruitières (Zhao et al., 2015 pour le poirier; Razouk et al., 2013a pour l'amandier; Pérez-Pastor et al., 2014 pour l'abricotier).

L'analyse statistique du LAI, à un seuil de probabilité de $5 \%$, en considérant chaque période à part, ne montre des différences significatives entre les traitements qu'à partir de la troisième période de la première année. Toutefois, le test de Dunnett montre que seul le $\mathrm{T} 0$ est significativement différent de $\mathrm{T} 1$, et ce pendant la deuxième année.

\subsubsection{Impact de la période sur l'évolution des paramètres étudiés}

À la lumière des résultats précédents, nous constatons que les organes de la tête du palmier dattier ont évolué pendant la période 
Tableau 8. Impact de la périodisation sur l'évolution des différents paramètres étudiés.

Table 8. Impact of periodization of the evolution of different parameters studied.

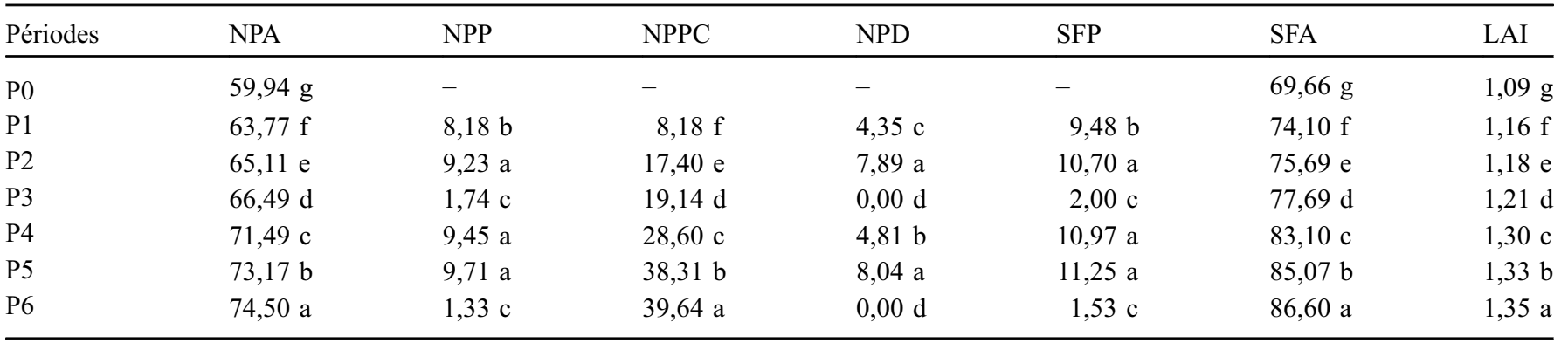

La même lettre dans la même colonne indique la non-signification des différences entre les périodes selon le test de Duncan.

NPA : nombre de palmes actives ; NPP : émission des palmes; NPPC : vitesse d'émission des palmes ; NPD : sénescence des palmes ; SFP : Surface foliaire des palmes; SFA: Surface foliaire active; LAI : indice foliaire.

Tableau 9. Synthèse des paramètres étudiés (significations statistiques).

Table 9. Summary of the parameters (statistical significance).

\begin{tabular}{lllllllll}
\hline Traitements & NPA & NPP & NPPC & NPD & NPDC & SFP & SFA \\
\hline T0 & $58,82 \mathrm{~b} *$ & $5,88 \mathrm{c} *$ & $22,76 \mathrm{~b} *$ & $4,71 \mathrm{a} *$ & $18,31 \mathrm{a}$ & $6,42 \mathrm{c} *$ & $64,57 \mathrm{~b} *$ & $1,01 \mathrm{~b}$ \\
T1 & $68,99 \mathrm{a}$ & $6,93 \mathrm{a}$ & $26,29 \mathrm{a}$ & $4,11 \mathrm{~b}$ & $15,81 \mathrm{c}$ & $8,09 \mathrm{ab}$ & $80,93 \mathrm{a}$ & $1,26 \mathrm{a}$ \\
T2 & $67,83 \mathrm{a}$ & $6,64 \mathrm{ab}$ & $25,47 \mathrm{a}$ & $3,94 \mathrm{~b}$ & $15,36 \mathrm{c}$ & $7,89 \mathrm{ab}$ & $80,85 \mathrm{a}$ & $1,26 \mathrm{a}$ \\
T3 & $67,11 \mathrm{a}$ & $6,42 \mathrm{~b}$ & $25,76 \mathrm{a}$ & $4,51 \mathrm{a}$ & $16,96 \mathrm{~b}$ & $7,42 \mathrm{~b}$ & $78,01 \mathrm{a}$ & $1,22 \mathrm{ab}$ \\
T4 & $70,31 \mathrm{a}$ & $6,64 \mathrm{ab}$ & $25,32 \mathrm{a}$ & $4,81 \mathrm{~b}$ & $14,75 \mathrm{c}$ & $7,57 \mathrm{ab}$ & $80,36 \mathrm{a}$ & $1,26 \mathrm{a}$ \\
T5 & $69,17 \mathrm{a}$ & $6,72 \mathrm{ab}$ & $25,44 \mathrm{a}$ & $3,97 \mathrm{~b}$ & $15,24 \mathrm{c}$ & $7,74 \mathrm{ab}$ & $79,72 \mathrm{a}$ & $1,25 \mathrm{a}$ \\
T6 & $72,60 \mathrm{a}$ & $7,03 \mathrm{a}$ & $26,43 \mathrm{a}$ & $4,07 \mathrm{~b}$ & $15,63 \mathrm{c}$ & $8,47 \mathrm{a}$ & $87,47 \mathrm{a}$ & $1,37 \mathrm{a}$ \\
TD/T1 & $*$ & $*$ & $*$ & $*$ & $*$ & $*$ & NS
\end{tabular}

La même lettre dans la même colonne indique la non-signification des différences entre les traitements selon le test de Duncan. NPA : nombre de palmes actives ; NPP : émission des palmes ; NPPC : vitesse d'émission des palmes ; NPD : sénescence des palmes ; NPDC : vitesse de sénescence des palmes; SFP : Surface foliaire des palmes; SFA : Surface foliaire active; LAI: indice foliaire TD/T1 : Test de Dunnett en prenant T1 comme référence (NS : non significatif; $*$ : significatif).

de l'essai (Tab. 8). Toutefois, cette évolution, remarquable en deuxième année, est moins importante en première année.

Un tel constat est évoqué par plusieurs études sur des espèces arboricoles telles que l'amandier, le pêcher (Razouk et al., 2013a), ou l'olivier (Dbara et al., 2011 ; Razouk et al., 2013b). Ce phénomène, couramment rencontré chez les arbres fruitiers, est dû essentiellement à la mobilisation des réserves au niveau du tronc et à l'importance de la zone racinaire (Fereres et Soriano, 2007 ; Katerji et Hallaire, 1984).

Chez le palmier, ce mécanisme d'adaptation n'a pas fait l'objet d'étude et les résultats obtenus ici pourraient constituer une première ébauche à développer et à enrichir par d'autres études.

Cette information est primordiale et ouvre un domaine très large concernant le comportement physiologique du dattier vis-à-vis de la pénurie d'eau tout au long de l'année à court, moyen et long termes, et ce en complément aux études morphologiques. Ceci pour mettre en évidence en premier lieu l'impact de l'eau sur le comportement physiologique du palmier et en second lieu pour comparer l'effet de la pénurie d'eau tout au long de l'année avec un déficit hydrique contrôlé à des moments spécifiques du cycle.

Nous avons aussi constaté que la période a un impact significatif sur l'évolution générale de la biomasse végétale du palmier dattier (Tab. 8). La période estivale (juillet-octobre) est la plus active, suivie par la période printanière (mars-juin); la période hivernale clôture le cycle avec une quasi-stabilité. Ces résultats confirment la majorité des références bibliographiques concernant la biologie de la croissance du palmier dattier (Munier, 1973 ; Sedra, 2003, 2012).

\subsection{6 Évolution moyenne des paramètres étudiés}

Le Tableau 9 synthétise l'évolution moyenne des variables étudiées, toutes périodes confondues. Excepté le LAI, les autres paramètres sont statistiquement différents $(\mathrm{P}<0,05)$ et les moyennes obtenues avec le traitement $\mathrm{T} 0$ 《agriculteur» sont différentes de celles du $\mathrm{T}_{1}(100 \% \mathrm{ETM})$.

La faiblesse des performances de la pratique de l'agriculteur est directement liée à la pratique de l'irrigation et à l'efficience du système existant.

L'agriculteur irrigue ses palmiers en goutte-à-goutte de la même façon qu'en irrigation gravitaire (doses importantes et faible fréquence, même parfois en période critique). Ceci engendre des pertes en eau énormes, qui dépassent la zone racinaire active et occasionnent par la même occasion des pertes d'éléments nutritifs. Cela diminue donc l'efficience de l'irrigation et de la fertilisation.

Le système d'irrigation localisée de l'agriculteur, mis en place depuis longtemps, s'est dégradé; de plus, le dimen- 
sionnement initial du réseau d'irrigation ne prévoyait pas l'extension de la surface plantée, qui dépasse aujourd'hui les capacités de la station de tête. Le résultat est une forte hétérogénéité de la distribution de l'eau.

Suite aux résultats de cette étude que nous avons discutés avec l'agriculteur, ce dernier a procédé au renouvellement de son système d'irrigation et au changement de ses modalités de gestion de l'irrigation en adoptant une fréquence d'arrosage raisonnée pour limiter les pertes.

\section{Conclusion}

L'évolution des paramètres de croissance et de développement de la biomasse végétale du palmier Majhoul, en conditions d'irrigation déficitaire, est généralement limitée pendant la première année de l'application des régimes de stress hydrique; cet impact a été ressenti significativement en seconde année. Le palmier dattier possède des capacités et des mécanismes de régulation de sa consommation d'eau selon les apports d'irrigation, qui évitent des pertes de rendement significatives pendant la première année de l'application du stress hydrique. Cela confirme et explique la présence évidente du palmier dattier même dans des situations où les conditions de ressources en eau sont très limitées.

La période la plus active du palmier dattier va de juillet à octobre. Durant cette période, on enregistre des changements et des réactions aux effets du stress hydrique.

Le traitement T6 (60-100-80\% ETM) est apparu le plus efficient puisqu'il a amélioré la croissance et le développement des palmes en produisant généralement des grandeurs supérieures au traitement le plus favorisé sur le plan hydrique, sans pour autant affecter les rendements.

L'interaction entre le régime hydrique et la période est toujours significative quelle que soit la variable considérée.

L'application de la technique de l'irrigation déficitaire contrôlée chez le palmier dattier est encore au stade expérimental et l'étude de son effet sur la régularité, la stabilité des rendements et la qualité des dattes à moyen et à long terme est nécessaire, par exemple pour mieux cerner la capacité du palmier à juguler des périodes de stress hydrique ponctuel. Des recherches sur les mécanismes qui régulent le rythme d'émission et de sénescence des feuilles permettraient de modéliser les processus de croissance en matière sèche, de répartition des assimilats entre organes (racines, stipes, feuilles et fruits) et d'élaboration du rendement en dattes.

\section{Références}

Allen RG, Pereira LS, Raes D, Smith M. 1998. Crop evapotranspiration: guidelines for computing crop water requirements. Irrigation and Drainage Paper 56 UN-FAO Rome.

Al-Khafaf S, Al Shiraqui R, Shabana H. 1998. Irrigation scheduling of palm trees in UAE. The 1st international conference on date palms, UAE University; Faculty of Agricultural Science.

Ballester C, Castel J, Abd El-Mageed TA, Castel JR, Intrigliolo DS. 2014. Long-term response of 'Clementina de Nules' citrus trees to summer regulated deficit irrigation. Agricultural Water Management 138: 78-84. Available from https://doi.org/10.1016/j.agwat.2014.03.003.

Ben Henia Y. 2012. Utilisation de la modélisation pour mieux concevoir les expérimentations relatives à la réponse à l'eau du palmier dattier dans la région d'Errachidia. Travail de fin d'études pour l'obtention du diplôme d'ingénieur en Agronomie, Option: management des productions végétales et de l'environnement. Maroc: IAV Hassan II, 84 p.

Carr MKV. 2012. The water relations and irrigation requirements of the date palm (Phoenix dactylifera L.): A review. Experimental Agriculture 49 (01): 91-113. DOI: 10.1017/ S0014479712000993.

Chalmers DJ, Mitchell PD, Van Heek L. 1981. Control of peach tree growth and productivity by regulated water supply, tree density, and summer pruning. Journal of the American Society for Horticultural Science 106: 307-312.

Dbara S, Ben Mimoun M, Hellali R. 2011. Réponse de l'olivier à la technique de dessèchement partiel des racines durant trois années consécutives d'essai. Tropicultura 29 (2): 70-74.

Djerbi M. 1988. Irrigation et drainage. In : Précis de phœniciculture. Document de la FAO : 55-69.

Doorenbos J, Pruitt WO. 1977. Crop water requirements. FAO Irrigation and Drainage Paper $\mathrm{N}^{\circ} 24$. Rome: FAO.

English MJ, Raja SN. 1996, Perspectives on deficit irrigation. Agricultural Water Management 32: 1-14.

FAO. 1980. Réponse des rendements à l'eau. Bulletin FAO de l'irrigation et du drainage $\mathrm{N}^{\circ} 33$. Rome: FAO.

Fereres E, Soriano MA. 2007. Deficit irrigation for reducing agricultural water use. Journal of Experimental Botany 58 (2): 147-159. DOI: 10.1093/jxb/erl165.

Gafar KY. 2010. Besoins en eau du palmier dattier en Émirats Arabes Unis. The Blessed Tree, Khalifa International Date palm Award 02 (03): 80-92.

Ismail SM, Al-Qurashi AD, Awad MA. 2014. Optimization of irrigation water use, yield, and quality of "Nabbutsaif" date palm under dry land conditions. Irrigation and Drainage 63: 29-37. DOI: $10.1002 /$ ird.1823.

Katerji N, Hallaire M. 1984, Les grandeurs de référence utilisables dans l'étude de l'alimentation en eau des cultures. Agronomie 4 (10): 999-1008.

Liebenberg PJ, Zaid A. 2002. Date palm irrigation. Chapter 7. In: Zaided A, ed. Date palm cultivation. Rome (Italy): FAO plan production and protection, paper No. 156.

MAPM. 2014. Situation de l'agriculture marocaine $\mathrm{N}^{\circ} 11$, édition de décembre 2014.

Mazahrih NTh, Al-Zubi Y, Ghnaim H, Lababdeh L, Ghananeem M, Ahmadeh HA. 2012. Determination actual evapotranspiration and crop coefficients of date palm trees (Phoenix dactylifera L.) in the Jordan Valley. American-Eurasian Journal of Agriculture and Environmental Science 12 (4): 434-443.

Munier P. 1973. Le palmier dattier - techniques agricoles et productions tropicales. France : Maisonneuve et Larousse, xxiv édition, $222 \mathrm{p}$.

ORMVA/TF. 2012. Monographie de la subdivision agricole de Goulmima. In : Monographie des subdivisions agricoles de l'office régional de mise en valeur agricole du tafilalet.

Pedrero F, Maestre-Valero JF, Mounzer O, Alarcón JJ, Nicolás E. 2014. Physiological and agronomic mandarin trees performance under saline reclaimed water combined with regulated deficit irrigation. Agricultural Water Management 146: 228-237. Available from https://doi.org/10.1016/j.agwat.2014.08.

Pereau-Leroy P. 1958. Le palmier dattier au Maroc. Ministère de l'Agriculture (Maroc) et Institut français de la recherche. Paris (France) : Fruit outre-mer, $142 \mathrm{p}$.

Pérez-Pastor A, Ruiz-Sánchez MaC, Domingo R. 2014. Effects of timing and intensity of deficit irrigation on vegetative and fruit growth of apricot trees. Agricultural Water Management 134: 110-118. Available from https://doi.org/10.1016/j.agwat.2013.12.007. 
Razouk R, Ibijbijen J, Kajji A, Karrou M. 2013a. Response of peach, plum and almond to water restrictions applied during slowdown periods of fruit growth. American Journal of Plant Sciences 4: 561570. DOI: 10.4236/ajps.2013.43073.

Razouk R, Ibijbijen J, Kajji A. 2013b. Optimal time of supplemental irrigation during fruit development of rainfed olive tree (Olea europaea, cv Picholine marocaine) in Morocco. American Journal of Experimental Agriculture 3 (4): 685-697.

Sabri A, Bouaziz A, Hammani A, Kuper M, Douaik A, Badraoui M. 2017. Effet de l'irrigation déficitaire contrôlée sur la productivité et l'efficience de l'utilisation de l'eau du palmier dattier cv Majhoul. Revue Marocaine des Sciences Agronomiques et Vétérinaires 5 (1): 23-31.
Sedra MyH. 2003. Le palmier dattier base de la mise en valeur des oasis du Maroc, techniques phoenicicoles et création d'oasis. Maroc : INRA, éditions 2003, 266 p.

Sedra MyH. 2012. Guide de phœniciculteur, mise en place et conduite des vergers phœnicicoles. Maroc : INRA, éditions 2012, $311 \mathrm{p}$.

Tardieu F. 2005. Plant tolerance to water deficit: physical limits and possibilities for progress. Comptes Rendus Géoscience 337 (1-2): 57-67. DOI: 10.1016/j.crte.2004.09.015.

Zhao Z, Wang W, Wu Y, Xu M, Huang X, Ma Y, et al. 2015. Leaf physiological responses of mature pear trees to regulated deficit irrigation in field conditions under desert climate. Scientia Horticulturae 187: 122-130. Available from https://doi.org/ 10.1016/j.scienta.2015.03.009.

Citation de l'article : Sabri A, Bouaziz A, Hammani A, Kuper M, Douaik A, Badraoui M. 2017. Effet de l'irrigation déficitaire contrôlée sur la croissance et le développement foliaire du palmier dattier (Phoenix dactylifera L.). Cah. Agric. $26: 55005$. 